especially as it relates to disease processes, to be explored in an efficient manner." Using a mouse model of a trait associated with a human disease, his group identifies regions of the mouse chromosome that control susceptibility to the disease-associated trait. Gene-expression profiling with the mouse microarrays is also done on target tissues from the mouse strains under study.

"The differentially expressed genes encoded within the identified chromosomal regions are the candidate genetic susceptibility loci," says Peltz. "We then analyse the function of the candidate genes (and pathways) in the biology related to the disease." A mouse model of osteoporosis was recently developed using this method, and an enzyme identified that affects bone mass and bone quality, which led to the development of a new potential therapeutic for treating osteoporosis.

IntegraGen's GenomeHIP technology platform takes a different microarray-based approach to discovering genes linked to complex disease traits in humans. The technology uses DNA chips for genomic comparisons of pairs of related individuals with the same disease to discover areas in which the genomes are identical — on the assumption that at least one of the relevant disease genes in such individuals will be identical in both. Crucial to IntegraGen's technology is the prior removal of most of the polymorphisms that differentiate the two genomes, cutting down the amount of genome that has to be searched. The company claims that its method is cheaper and faster than conventional methods of gene identification, such as linkage analysis using microsatellite markers. Unlike such methods, which can require several generations of many different families, the GenomeHIP technology can identify potential disease genes with high statistical significance in a relatively small sample of patients (50-150 pairs of related individuals with the same trait) in less than nine months.

Using this platform, IntegraGen recently identified a mutant G-proteincoupled receptor that is strongly associated with obesity in various populations. "The most frequent mutation observed was demonstrated to impair the signalling through this receptor by its natural ligands," says Mous. "The normal function of this receptor, which is predominantly expressed in the gut, is to transmit an anorexic signal, which could be impaired in patients expressing the mutant form."

The advent of the complete human-gene chips is generally welcomed as a important tool. But Fitzgerald cautions that, like most molecular tools, gene chips have limitations of which one must be aware. "Experimental design with an eye towards rigorous statistical analysis is crucial," he says. "Gene chips produce a large number of individual gene patterns and if one is asking a computer program to find patterns in the data, it will find patterns. If the experiments were not correctly designed and controlled, those patterns can be quite misleading."

$\mathrm{He}$ also stresses the importance of remembering that in general, gene chips measure correlative rather than causative events. Andrea Gnirke of Xantos Biomedicine in Munich, Germany, also makes the point that the chips provide an analytical challenge. "Chip data can only be correlative data and implicate an enormous effort for data analysis, and it is difficult to sort out the 'good' targets that have causative effects in diseases," she says.

And because much cellular regulation occurs at the level of proteins, "increased transcript production as measured by gene chips does not always correlate with production of protein, and even if more protein is produced it may not be active because it requires post-translational modification or relocalization within a cell", cautions Fitzgerald. Jean-Jacques Yarmoff of Hybrigenics agrees: "Modifications of proteins lead to an additional level of complexity that is not necessarily addressable by humangene chips."

\section{Tackling the 'interactome'}

Because interactions between proteins are key to their function, determining the pathways within which a potential drug target

\title{
GETTING UP SPEED
}

Multiplexing in highly automated systems is one way of speeding up the assays used for target identification. Bio-Rad in Hercules, California, offers Bio-Plex, a multiplex analysis system that allows the simultaneous analysis of up to 100 different biomolecules (proteins, peptides or nucleic acids) in a single microplate well. Bio-Plex integrates assay kits, software, calibration and validation tools, and instrumentation into a complete system. The Bio-

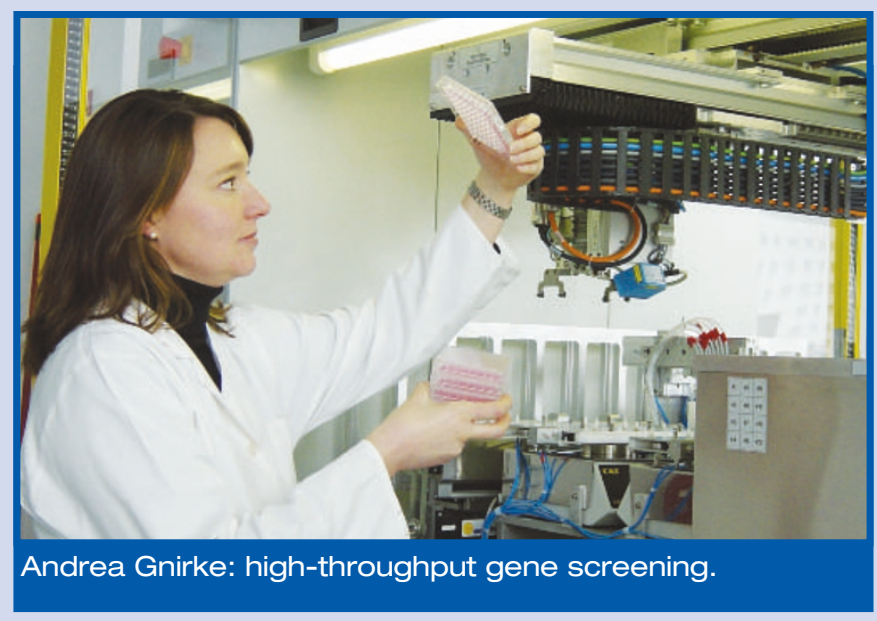

Plex suspension array and workstation uses three main technologies: a family of fluorescence-tagged microspheres from Luminex in Austin, Texas; a flow cytometer that uses two lasers to measure biochemical reactions occurring on the surface of the microspheres; and a high-speed digital signal processor to manage the fluorescent output.

In its target-identification programme, Xantos Biomedicine of Munich, Germany, uses high-throughput cellular screening assays to study gene function in parallel for thousands of genes. For whole-genome screening, its proprietary XantoPrep and XantoScreen high-throughput system screens complementary DNA libraries by overexpression in transient transfection systems, enabling Xantos to carry out functional analysis on up to 100,000 different cDNAs per month. "Genes or proteins are selected by their ability to directly influence physiologically relevant processes or pathways in cellular assays, leading to a very small number of relevant hits compared with expression-profiling studies, for example," says Andrea Gnirke, senior scientist for expression analysis at Xantos. These methods have been used to identify secreted proteins influencing the differentiation of fat cells, which play a major role in the development of insulin resistance, and which Xantos hopes could have potential for the treatment of obesity-related type II diabetes. An interesting future direction for Xantos, along with many other companies, includes RNA interference (RNAi). "Xantos is implementing a confocal high-content imaging screening system and aims to integrate RNAi technology for high-throughput application," says Gnirke. 